VOL. 63 (2001) [75-81]

\title{
THE FIXED POINT PROPERTY AND NORMAL STRUCTURE FOR SOME B-CONVEX BANACH SPACES
}

\author{
Jesús García-Falset, Enrique Llorens-Fuster \\ AND EVA M. MazCuñáN-NaVARRo
}

\begin{abstract}
We give a sufficient condition for normal structure more general than the well known $\varepsilon_{0}(X)<1$. Moreover we obtain sufficient conditions for the fixed point property for some B-convex Banach spaces.
\end{abstract}

\section{INTRODUCTION}

Let $(X,\|\cdot\|)$ be a real Banach space, and let $C$ be a nonempty subset of $X$. A mapping $T: C \rightarrow X$ is said to be nonexpansive if $\|T(x)-T(y)\| \leqslant\|x-y\|$ for all $x, y \in C$. We say that $(X,\|\cdot\|)$ has the weak fixed point property, if every nonexpansive selfmapping $T$ on any weakly compact convex subset $C$ of $X$ has a fixed point.

It is well known that the weak fixed point property holds for Banach spaces with certain geometrical properties. Among such properties, weak normal structure is maybe the most widely studied (see [7]). Sufficient conditions for the weak fixed point property or weak normal structure have been given in terms of the characteristic of convexity $\varepsilon_{0}(X)$.

For example, it is well known that Banach spaces with $\varepsilon_{0}(X)<1$ enjoy both normal structure and superreflexivity [7]. However, it remains unknown whether the weak fixed point property holds for every uniformly nonsquare Banach space $X$ (that is, $\varepsilon_{0}(X)<2$ ). Some partial affirmative answers to this question have been established in $[3,12]$.

In a recent paper [8] Jiménez-Melado introduced a geometrical coefficient $\widetilde{\varepsilon_{0}}(X)$ which is a natural generalisation of the classical characteristic of convexity $\varepsilon_{0}(X)$. He improved the result of [3], proving that a Banach space $X$ with $\widetilde{\varepsilon_{0}}(X)<2$ has the weak fixed point property whenever $X$ has the (WORTH) property defined by Sims in [11]. If $\widetilde{\varepsilon_{0}}(X)<2$ then $X$ is uniformly nonoctahedral.

In this paper we generalise the classical sufficient condition for normal structure $\varepsilon_{0}(X)<1$, to $\widetilde{\varepsilon_{0}}{ }^{k}(X)<1$ where $\widetilde{\varepsilon_{0}}{ }^{k}(X)$ is an extension to higher finite dimensions of the coefficient $\widetilde{\varepsilon_{0}}(X)={\widetilde{\varepsilon_{0}}}^{2}(X)$, which has a three dimensional character. Moreover we obtain

Received 30th March, 2000

The authors were partially supported by D.G.E.S. PB96-1338-C02-02 and P.S.F.P.U. AP98, Ministerio Educación y Cultura, Spain.

Copyright Clearance Centre, Inc. Serial-fee code: 0004-9727/01 SA2.00+0.00. 
sufficient conditions for the weak fixed point property for spaces $X$ with $\widetilde{\varepsilon_{0}}{ }^{k}(X)<2$. Such conditions are different to the (WORTH) property.

\section{DEFINITIONS AND PRELIMINARIES}

Let $(X,\|\cdot\|)$ be a Banach space. Let $B_{X}$ and $S_{X}$ denote the closed unit ball and the unit sphere respectively.

The following definitions and results will be used throughout this paper. The function $\delta_{X}:[0,2] \rightarrow[0,1]$ given by

$$
\delta_{X}(\varepsilon):=\inf \left\{1-\left\|\frac{1}{2}(x+y)\right\|: x, y \in B_{X},\|x-y\| \geqslant \varepsilon\right\},
$$

is Clarkson's modulus of convexity of $(X,\|\cdot\|)$. The characteristic of converity of $X$ is defined by $\varepsilon_{0}(X):=\sup \left\{\varepsilon \in[0,2]: \delta_{X}(\varepsilon)=0\right\}$.

A convex set $C \subset X$ is said to have normal structure if, for each nonempty closed convex bounded subset $K$ of $C$, which is not a singleton, there exists at least one nondiametral point $x \in K$, that is, with $r(x, K):=\sup \{\|x-y\|: y \in K\}<\operatorname{diam}(K)$. Similarly, the convex set $C$ has weak normal structure provided that each weakly compact convex nontrivial subset $K$ of $C$ contains a nondiametral point.

Following Sims, [10], we shall say that $(X,\|\cdot\|)$ has the weak uniform Kadec-Klee property whenever there exist $\varepsilon \in(0,1)$ and $\delta \in(0,1)$, such that $\operatorname{dist}(0, C) \leqslant 1-\delta$ for every weakly compact convex subset $C$ of $B_{X}$ with $\gamma(C)>\varepsilon$, where $\gamma(C)$ is the separation measure of noncompactness defined by

$$
\gamma(C):=\sup \left\{\inf _{n \neq m}\left\|x_{m}-x_{n}\right\|:\left(x_{n}\right) \text { a sequence inC }\right\} .
$$

If moreover $X$ is reflexive, then we say that $X$ is weakly nearly uniformly convex.

Following [4], given a measure of noncompactness $\mu$, we define the modulus of convexity of $X$ with respect to $\mu$ to be the function $\Delta_{\mu}:\left[0, \mu\left(B_{X}\right)\right) \rightarrow[0,1]$ given by

$$
\Delta_{\mu}(\varepsilon):=\inf \left\{1-\operatorname{dist}(0, A): A \subset B_{X}, A \text { is convex and } \mu(A) \geqslant \varepsilon\right\},
$$

and we define the coefficient of convexity of $X$ with respect to $\mu$ to be the number $\varepsilon_{1}(\mu):=$ $\sup \left\{\varepsilon>0: \Delta_{\mu}(\varepsilon)=0\right\}$. Moreover it is shown in [4] that the Banach space $(X,\|\cdot\|)$ has weak normal structure if $\Delta_{\mu}(1) \neq 0$. (In particular this happen if $\varepsilon_{1}(\mu)<1$.)

The Banach space $(X,\|\cdot\|)$ has the WORTH property if

$$
\lim _{n \rightarrow \infty}\left|\left\|x_{n}-x\right\|-\left\|x_{n}+x\right\|\right|=0
$$

for all $x \in X$ and for all weakly null sequences $\left(x_{n}\right)$ in $X$. 
A sequence $\left(x_{n}\right)$ in a Banach space $X$ is called a Schauder basis of $X$ if for every $x \in X$ there exists a unique sequence of scalars $\left(a_{n}\right)$ such that $x=\sum_{n=1}^{\infty} a_{n} x_{n}$. A Schauder basis is called an unconditional basis if and only if the constant

$$
\lambda=\sup \left\{\left\|\sum_{n=1}^{\infty} \varepsilon_{n} a_{n} x_{n}\right\|:\left\|\sum_{n=1}^{\infty} a_{n} x_{n}\right\|=1, \varepsilon_{i}= \pm 1\right\}
$$

is finite. When $\left(x_{n}\right)$ is an unconditional basis, we define the following constant:

$$
c=\sup \left\{\left\|P_{F}\right\|: F \subset \mathbb{N}\right\}
$$

where $P_{F}\left(\sum_{n=1}^{\infty} a_{n} x_{n}\right)=\sum_{n \in F} a_{n} x_{n}$. We say that $\left(x_{n}\right)$ is a suppression unconditional when $c=1$.

We say that $\left(x_{n}\right)$ is a strongly bimonotone Schauder basis if $\left\|P_{F}\right\|=\left\|I-P_{F}\right\|=1$ for every segment $F=[a, b]:=\{n \in \mathbb{N}: a \leqslant n \leqslant b\}$.

A Banach space $X$ has the weak Banach-Saks property if every weak null sequence $\left(x_{n}\right)$ contains a subsequence $\left(x_{n_{k}}\right)$ such that $\left\{(1 / n) \sum_{i=1}^{n} x_{n_{i}}\right\}$ is norm-convergent.

In [2] the author proved the following result:

LemMa 1. Suppose $X$ has a strongly bimonotone basis $\left(x_{n}\right)$. Then $X$ has the weak fixed point property whenever $X$ has the weak Banach-Saks property.

Following Giesy and James [6], for $n \geqslant 2$, a Banach space is called uniformly non- $\ell_{1}^{n}$ if there exists $\varepsilon>0$ such that there does not exist a subset $\left\{x_{1}, \ldots, x_{n}\right\}$ of the unit ball $B_{X}$ with

$$
\left\|x_{1} \pm x_{2} \pm \cdots \pm x_{n}\right\|>n(1-\varepsilon)
$$

for all choices of signs. We say that $X$ is B-convex if it is uniformly non- $\ell_{1}^{n}$ for some $n \geqslant 2$.

\section{SUFFICIENT CONDITIONS FOR NORMAL STRUCTURE}

Let $(X,\|\cdot\|)$ be a Banach space and let $k \in \mathbb{N}$.

Denote by $s_{k}(X)$ the supremum of the set of numbers $\varepsilon \in[0,2]$ for which there exist points $x_{1}, \ldots, x_{k+1}$ in $B_{X}$ with $\min \left\{\left\|x_{i}-x_{j}\right\|: i \neq j\right\} \geqslant \varepsilon$.

Define the function $\widetilde{\delta}^{k}:\left[0, s_{k}(X)\right) \longrightarrow[0,1]$ by

$$
\tilde{\delta}^{k}(\varepsilon):=\inf \left\{1-\left\|\frac{x_{1}+\cdots+x_{k+1}}{k+1}\right\|: x_{i} \in B_{X}, i=1, \ldots, k+1, \min _{i \neq j}\left\|x_{i}-x_{j}\right\| \geqslant \varepsilon\right\}
$$

and let ${\widetilde{\varepsilon_{0}}}^{k}(X)$ be the number $\widetilde{\varepsilon}_{0}^{k}(X):=\sup \left\{\varepsilon \in\left[0, s_{k}(X)\right): \tilde{\delta}^{k}(\varepsilon)=0\right\}$. 
REMARK 1. Notice that ${\widetilde{\varepsilon_{0}}}^{1}(X)=\varepsilon_{0}(X)$, the usual characteristic of convexity, and $\widetilde{\varepsilon_{0}}(X)=\widetilde{\varepsilon_{0}}(X)$ where $\widetilde{\varepsilon_{0}}(X)$ is the characteristic of convexity introduced by Jiménez Melado in [8].

The following proposition relates the characteristics of convexity defined above for different dimensions.

Proposition 1. For any $k \in \mathbb{N}$,

$$
{\widetilde{\varepsilon_{0}}}^{k+1}(X) \leqslant{\widetilde{\varepsilon_{0}}}^{k}(X)
$$

Moreover,

$$
\widetilde{\varepsilon_{0}}(X) \leqslant \varepsilon_{0}(X) \leqslant 2 \widetilde{\varepsilon_{0}}(X) .
$$

Proof: Fix $k \in \mathbb{N}$. Let $\varepsilon \in\left[0, s_{k+1}(X)\right)$. For any $x_{1}, \ldots, x_{k+2}$ in $B_{X}$ with $\min _{i \neq j} \| x_{i}-$ $x_{j} \| \geqslant \varepsilon$

$$
\left\|\frac{x_{1}+\cdots+x_{l-1}+x_{l+1}+\cdots+x_{k+2}}{k+1}\right\| \leqslant 1-\tilde{\delta}^{k}(\varepsilon) \quad \text { for } l=1, \ldots, k+2
$$

and consequently,

$$
\begin{aligned}
\left\|\frac{x_{1}+\cdots+x_{k+2}}{k+2}\right\| & =\left\|\frac{1}{k+2} \sum_{l=1}^{k+2} \frac{x_{1}+\cdots+x_{l-1}+x_{l+1}+\cdots+x_{k+2}}{k+1}\right\| \\
& \leqslant \frac{1}{k+2} \sum_{l=1}^{k+2}\left\|\frac{x_{1}+\cdots+x_{l-1}+x_{l+1}+\cdots+x_{k+2}}{k+1}\right\| \\
& \leqslant 1-\tilde{\delta}^{k}(\varepsilon) .
\end{aligned}
$$

So we have that for any $\varepsilon \in\left[0, s_{k+1}(X)\right), \quad \tilde{\delta}^{k+1}(\varepsilon) \geqslant \tilde{\delta}^{k}(\varepsilon)$, and therefore ${\widetilde{\varepsilon_{0}}}^{k+1}(X) \leqslant$ $\tilde{\varepsilon}_{0}^{k}(X)$.

On the other hand, given $\varepsilon>0$ consider $x, y \in B_{X}$, with $\|x-y\| \geqslant \varepsilon$. Let $z=$ $(x+y) / 2$. We have that $\|x-z\|=\|y-z\|=\|x-y\| / 2 \geqslant \varepsilon / 2$.

Consequently, $1-\|(x+y) / 2\|=1-\|(x+y+z) / 3\| \geqslant \tilde{\delta}(\varepsilon / 2)$. Therefore, $\delta(\varepsilon) \geqslant$ $\tilde{\delta}(\varepsilon / 2)$ for all $\varepsilon>0$, and then $\varepsilon_{0}(X) \leqslant 2 \widetilde{\varepsilon_{0}}(X)$.

REMARK 2. In [8] the author asks whether a Banach space $X$ with $\widetilde{\varepsilon_{0}}(X)<2$ is reflexive. From the above Proposition it is clear that if $\widetilde{\varepsilon_{0}}(X)<1$ then $X$ is superreflexive.

In some cases, the inequality is strict. To see this we consider the following example.

EXAMPLE 1. Let $\ell_{\infty}^{(2)}$ be the Banach space $\mathbf{R}^{2}$ endowed with the supremum norm. It is not difficult to see that for each $k \in \mathbb{N}$,

$$
\widetilde{\varepsilon}_{0}^{k}\left(\ell_{\infty}^{(2)}\right)=\frac{2}{k}
$$


The following result shows the relation between ${\widetilde{\varepsilon_{0}}}^{k}(X)$ and the coefficient of convexity of $X$ with respect to the measure of noncompactness $\gamma$.

Proposition 2. Let $X$ a Banach space with $\widetilde{\varepsilon}_{0}^{k}(X)<1$ for some $k \in \mathbb{N}$. Then also $\varepsilon_{1}(\gamma)<1$.

PRoof: Since ${\widetilde{\varepsilon_{0}}}^{k}(X)<1$ there exists $\varepsilon \in(0,1)$ such that $\widetilde{\delta}^{k}(1-\varepsilon)>0$. Suppose, seeking a contradiction, that $\varepsilon_{1}(\gamma) \geqslant 1$. Then $\Delta_{\gamma}(1-\varepsilon / 2)=0$, and consequently there exists $C \subseteq B_{X}$ convex with $\gamma(C) \geqslant 1-\varepsilon / 2$ and $1-\operatorname{dist}(0, C)<\widetilde{\delta}^{k}(1-\varepsilon)$. Now, since $\gamma(C)>1-\varepsilon$ there exists a sequence $\left(x_{n}\right)$ in $C$ with the property $\inf _{n \neq m}\left\|x_{n}-x_{m}\right\|>1-\varepsilon$.

In particular $x_{1}, \ldots, x_{k+1} \in B_{X}$ and $\min _{\substack{1 \leqslant i \leqslant k+1 \\ i \neq j}}\left\|x_{i}-x_{j}\right\|>1-\varepsilon$, from which we obtain the following contradiction which completes the proof:

$$
\begin{aligned}
\tilde{\delta}^{k}(1-\varepsilon) & \leqslant 1-\left\|\frac{x_{1}+\cdots+x_{k+1}}{k+1}\right\| \\
& \leqslant 1-\operatorname{dist}(0, C) \\
& <\tilde{\delta}^{k}(1-\varepsilon) .
\end{aligned}
$$

It is clear from Proposition 2 and the above mentioned results of [4] that we have the following.

ThEOREM 1. If $X$ is a Banach space such that ${\widetilde{\varepsilon_{0}}}^{k}(X)<1$ for some $k \in \mathbb{N}$, then

(a) $X$ is weakly nearly uniformly convex.

(b) $X$ is reflexive and has normal structure.

The well known result that $\varepsilon_{0}(X)<1$ implies $X$ has normal structure is a strictly particular case of Theorem 1.

EXAMPLE 2. In the classical real sequence space $\left(\ell_{2},\|\cdot\|\right)$ we can consider the equivalent norm $|\cdot|_{\beta}$, given by $|x|_{\beta}:=\max \left\{\|x\|_{2}, \beta\|x\|_{\infty}\right\}$. Let $E_{\beta}:=\left(\ell_{2},|\cdot|_{\beta}\right)$. Suppose that $\tilde{\varepsilon}_{0}\left(E_{\beta}\right) \geqslant 1$. Then $\bar{\delta}(1-(1 / n))=0$ for all $n \in \mathbb{N}$, and consequently there exist sequences $\left(x_{n}^{i}\right)_{n}$ in $B_{E_{\beta}},(i=1,2,3)$, such that for all $n \in \mathbb{N}, \min \left\{\left|x_{n}^{i}-x_{n}^{j}\right|_{\beta}: i \neq j\right\} \geqslant 1-1 / n$ and

$$
1-\left|\frac{1}{3}\left(x_{n}^{1}+x_{n}^{2}+x_{n}^{3}\right)\right|_{\beta} \leqslant \frac{1}{n} .
$$

Since the norm $\|\cdot\|$ is the Euclidean one and for all $v \in \ell_{2},\|v\| \leqslant|v|_{\beta} \leqslant \beta\|v\|$, we have that

$$
\begin{aligned}
\frac{1}{\beta^{2}}\left|x_{n}^{1}+x_{n}^{2}+x_{n}^{3}\right|_{\beta}^{2} & \leqslant\left\|x_{n}^{1}+x_{n}^{2}+x_{n}^{3}\right\|^{2} \\
& =3 \sum_{i=1}^{3}\left\|x_{n}^{i}\right\|^{2}-\sum_{i<j}\left\|x_{n}^{i}-x_{n}^{j}\right\|^{2} \\
& \leqslant 3 \sum_{i=1}^{3}\left|x_{n}^{i}\right|_{\beta}^{2}-\frac{1}{\beta^{2}} \sum_{i<j}\left|x_{n}^{i}-x_{n}^{j}\right|_{\beta}^{2}
\end{aligned}
$$


for all $n \in \mathbb{N}$. So we have

$$
\frac{9}{\beta^{2}}\left(1-\frac{1}{n}\right)^{2} \leqslant 9-\frac{3}{\beta^{2}}\left(1-\frac{1}{n}\right)^{2}
$$

for all $n \in \mathbb{N}$, that is, $\beta \geqslant \sqrt{4 / 3}$. Thus, $\tilde{\varepsilon}_{0}\left(E_{\beta}\right)<1$ for $\beta<\sqrt{4 / 3}$. Nevertheless, it is well known (see $[7, \mathrm{p} .58]$ ) that, for $\beta \leqslant \sqrt{2}, \varepsilon_{0}\left(E_{\beta}\right)=2 \sqrt{\beta^{2}-1}$. So, if $\beta \in((\sqrt{5} / 2), \sqrt{4 / 3})$ we have that $\widetilde{\varepsilon_{0}}\left(E_{\beta}\right)<1<\varepsilon_{0}\left(E_{\beta}\right)$.

REMARK 3. The Bynum space $\ell_{p, \infty}, 1<p<\infty$, (see [7]) fails to have normal structure, so by Theorem 1 we see that ${\widetilde{\varepsilon_{0}}}^{k}(X) \geqslant 1$ for all $k$. But, by Proposition 1 we have ${\widetilde{\varepsilon_{0}}}^{k}\left(\ell_{p, \infty}\right) \leqslant \varepsilon_{0}\left(\ell_{p, \infty}\right)=1$ for all $k$. So $\widetilde{\varepsilon_{0}}\left(\ell_{p, \infty}\right)=1$ for all $k \in \mathbb{N}$. This shows that the assumption ${\widetilde{\varepsilon_{0}}}^{k}\left(\ell_{p, \infty}\right)<1$ in Theorem 1 is sharp.

\section{THE FIXED POINT PROPERTY FOR SOME UNIFORMLY NON- $\ell_{1}^{n}$ SPACES}

THEOREM 2. If $X$ is a Banach space with a strongly bimonotone basis and $\widetilde{\varepsilon_{0}}(X)<2$ for some $k \in \mathbb{N}$ then $X$ has the weak fixed point property.

Proof: By means of Lemma 1 it suffices to prove $X$ has the weak Banach-Saks property provided $\widetilde{\varepsilon}_{0}^{k}(X)<2$ for some $k \in \mathbb{N}$.

Suppose $X$ is a Banach space with $\widetilde{\varepsilon}_{0}^{k}(X)<2$, but $X$ fails to be uniformly non$\ell_{1}^{k+1}$. Then for all $\varepsilon>0$ there exist $x_{1}(\varepsilon), \ldots, x_{k+1}(\varepsilon)$ in $B_{X}$ such that for any choice of the " + " and "-" signs

$$
\left\|x_{1}(\varepsilon) \pm \cdots \pm x_{k+1}(\varepsilon)\right\|>(k+1)(1-\varepsilon) .
$$

Now, for fixed $i, j \in\{1, \ldots, k+1\}$, we have from (1) that for all $\varepsilon>0$

$$
\begin{aligned}
\left\|x_{i}(\varepsilon)-x_{j}(\varepsilon)\right\| & \geqslant\left\|\left[\sum_{l \in\{1, \ldots, k+1\}-\{j\}} x_{l}(\varepsilon)\right]-x_{j}(\varepsilon)\right\|-\sum_{l \in\{1, \ldots, k+1\}-\{i, j\}}\left\|x_{l}(\varepsilon)\right\| \\
& >(k+1)(1-\varepsilon)-(k-1) \\
& =2-(k+1) \varepsilon
\end{aligned}
$$

Therefore, using (1) once more, for all $\varepsilon>0$

$$
\tilde{\delta}^{k}(2-(k+1) \varepsilon) \leqslant 1-\left\|\frac{x_{1}(\varepsilon)+\cdots+x_{k+1}(\varepsilon)}{k+1}\right\| \leqslant 1-(1-\varepsilon)=\varepsilon .
$$

Since ${\widetilde{\varepsilon_{0}}}^{k}(X)<2$ we can choose $\eta \in\left(0,2-{\widetilde{\varepsilon_{0}}}^{k}(X)\right)$.

Consider $\rho:=\min \left\{\tilde{\delta}^{k}(2-\eta) / 2, \eta /(k+1)\right\}>0$. Since the function $\tilde{\delta}^{k}(\cdot)$ is nondecreasing

$$
\tilde{\delta}^{k}(2-(k+1) \rho) \geqslant \tilde{\delta}^{k}(2-\eta)>\rho
$$

which contradicts (2). Thus, if ${\widetilde{\varepsilon_{0}}}^{k}(X)<2, X$ is uniformly non- $\ell_{1}^{k+1}$. Now, from [9] (see also [1]), $X$ has the weak Banach-Saks property. 
REMARK 4. It is well known (see [5]) that a B-convex Banach space is reflexive provided that either it has an unconditional basis or it is a Banach lattice. Thus, if $\overline{\varepsilon_{0}}(X)<2$ and $X$ has an unconditional basis then $X$ is reflexive. This is a partial answer to the question raised by Jiménez-Melado in [8]. Consequently, if $X$ is a Banach space with a suppression unconditional basis such that $\widetilde{\varepsilon_{0}}{ }^{k}(X)<2$ for some $k \in \mathbb{N}$, then $X$ has the fixed point property.

REMARK 5. It is easy to see that the space $\ell_{p, \infty}(1<p<\infty)$ has a suppression unconditional basis but it fails to have the (WORTH) property. Therefore, it is an example for which Theorem 2 applies, but it is out of the scope of the result of [8]. On the other hand, since any nonseparable Hilbert space has the (WORTH) property, Theorem 2 and the result of $[8]$ are different.

\section{REFERENCES}

[1] B. Beauzamy, 'Banach-Saks properties and spreading models', Math. Scand. 44 (1979), 357-384.

[2] J. García Falset, 'Basis and fixed points for nonexpansive mappings', Rad. Mat. 8 (1992), 67-75.

[3] J. García Falset, 'Fixed point property in Banach spaces whose characteristic of convexity is less than 2', J. Austral. Math. Soc 54 (1993), 169-173.

[4] J. Garcia-Falset, A. Jiménez-Melado and E. Lloréns-Fuster, 'Measures of noncompactness and normal structure in Banach spaces', Studia Math. 110 (1994), 1-8.

[5] D.P. Giesy, 'B-convexity and reflexivity', Israel J. Math. 15 (1973), 430-436.

[6] D.P. Giesy and R.C. James, 'Uniformly non- $\ell(1)$ and B-convex Banach spaces', Studia Math. 48 (1973), 61-69.

[7] K. Goebel and W.A. Kirk, Topics in metric fixed point theory, Cambridge Stud. Adv. Math. 28 (Cambridge, 1990).

[8] A. Jiménez Melado, 'The fixed point property for some uniformly nonoctahedral Banach spaces', Bull. Austral. Math. Soc. 59 (1999), 361-367.

[9] H.P. Rosenthal, 'Weakly independent sequences and the Banach-Saks property', (Proceedings of the Durham Symposium on the relations between infinite-dimensional and finite-dimensional convexity. Durham-July, 1975, p.26).

[10] B. Sims, 'Fixed points of nonexpansive maps on weak and weak*-compact sets', (Queen's Univ. of Kingston Lecture notes, 1982).

[11] B. Sims, 'Orthogonality and fixed points of nonexpansive maps', Proc. Centre Math. Anal. Austral. Nat. Univ. 20 (1988), 178-186.

[12] B. Sims, 'A class of spaces with weak normal structure', Bull. Austral. Math. Soc. 49 (1994), 523-528.

Departamento de Análisis Matemático

Facultad de Matemáticas

Doctor Moliner 50

46100 Burjasot, Valencia

Spain 